\title{
Generic Properties of Continuous Iterated Function Systems with Place Dependent Probabilities
}

by

\author{
Tomasz BIELACZYC \\ Presented by Stanisław KWAPIEŃ
}

Summary. It is shown that for a typical continuous learning system defined on a compact convex subset of $\mathbb{R}^{n}$ the Hausdorff dimension of its invariant measure is equal to zero.

1. Introduction. Generic properties of Markov operators were studied in $[1,5,7,8,10]$. In [1] it was proved that for a typical finite iterated function system with place dependent probabilities satisfying the average contractivity condition the Hausdorff dimension of its stationary distribution is equal to zero.

In this paper we prove an analogous result for nonexpansive continuous iterated function systems with place dependent probabilities satisfying the condition $\int_{0}^{K}|p(t, x)-p(t, y)| d t \leq c_{p}\|x-y\|$.

Continuous iterated function systems with place dependent probabilities were studied by A. Lasota and M. C. Mackey in [4]. They were interested in biological interpretation of such systems. They applied criteria for asymptotic stability of such systems to describe stability of cell structures.

Continuous iterated function systems were also studied by T. Szarek [7] who showed that a typical system is asymptotically stable and its stationary distribution is singular. The proofs of Lemma 3.6 and Theorem 4.1 base on the approximation method used by T. Szarek (see Lemma 3.5 and Theorem 3.1).

2000 Mathematics Subject Classification: Primary 60J25, 26A18; Secondary 37A30, $28 \mathrm{D} 05$.

Key words and phrases: Markov operators, iterated function systems, Hausdorff dimension. 
The organization of the paper is as follows. In Section 2 we introduce the definitions and notation. Section 3 contains auxiliary lemmas which are used in proving the main result of the paper. The main theorem is proved in Section 4.

2. Preliminaries. Let $X \subset \mathbb{R}^{k}$ be a compact convex set and let $\mathcal{B}(X)$ denote the $\sigma$-algebra of all Borel subsets of $X$.

Throughout this paper $B(x, r)$ stands for the open ball with center at $x \in X$ and radius $r>0$. Given a set $A \subset X$ and a number $r>0$ we denote by $B(A, r)$ the open $r$-neighbourhood of the set $A$ in $X$.

We denote by $\mathcal{M}$ the family of all finite Borel measures on $X$, and by $\mathcal{M}_{1}$ the space of all $\mu \in \mathcal{M}$ such that $\mu(X)=1$. The elements of $\mathcal{M}_{1}$ are called distributions.

Let $\mathcal{M}_{\mathrm{s}}$ be the space of all finite signed Borel measures on $X$. For every $l \geq 1$ we introduce the Fortet-Mourier norm

$$
\|\mu\|_{l}=\sup \left\{|\langle f, \mu\rangle|: f \in F_{l}\right\},
$$

where

$$
\langle f, \mu\rangle=\int_{X} f(x) \mu(d x)
$$

and $F_{l}$ is the space of all continuous functions $f: X \rightarrow \mathbb{R}$ such that $\sup _{x \in X}|f(x)| \leq 1$ and $|f(x)-f(y)| \leq l\|x-y\|$ for $x, y \in X$. Since the convergence in the Fortet-Mourier norm is equivalent to the weak convergence (see [2]), the norms $\|\cdot\|_{l_{1}}$ and $\|\cdot\|_{l_{2}}$ for $l_{1}, l_{2} \geq 1$ are equivalent.

An operator $P: \mathcal{M} \rightarrow \mathcal{M}$ is called a Markov operator if it is positive linear and preserves the norm. An operator $P: \mathcal{M} \rightarrow \mathcal{M}$ is called nonexpansive in the norm $\|\cdot\|_{l}, l \geq 1$, if

$$
\left\|P \mu_{1}-P \mu_{2}\right\|_{l} \leq\left\|\mu_{1}-\mu_{2}\right\|_{l} \quad \text { for } \mu_{1}, \mu_{2} \in \mathcal{M}_{1} .
$$

A measure $\mu \in \mathcal{M}$ is called stationary or invariant if $P \mu=\mu$. A Markov operator $P$ is called asymptotically stable if there exists a stationary distribution $\mu_{\star}$ such that

$$
\lim _{n \rightarrow \infty}\left\langle f, P^{n} \mu\right\rangle=\left\langle f, \mu_{\star}\right\rangle \quad \text { for } \mu \in \mathcal{M}_{1}, f \in C(X)
$$

(here $C(X)$ stands for the space of all continuous functions $f: X \rightarrow \mathbb{R}$ ).

For $A \subset X$ and $s, \delta>0$ define

$$
\mathcal{H}_{\delta}^{s}(A)=\inf \left\{\sum_{i=1}^{\infty}\left(\operatorname{diam} U_{i}\right)^{s}: A \subset \bigcup_{i=1}^{\infty} U_{i}, \operatorname{diam} U_{i} \leq \delta\right\}
$$

and

$$
\mathcal{H}^{s}(A)=\lim _{\delta \rightarrow 0} \mathcal{H}_{\delta}^{s}(A)
$$


The value

$$
\operatorname{dim}_{\mathrm{H}} A=\inf \left\{s>0: \mathcal{H}^{s}(A)=0\right\}
$$

is called the Hausdorff dimension of the set $A$.

The Hausdorff dimension of a measure $\mu \in \mathcal{M}_{1}$ is defined by the formula

$$
\operatorname{dim}_{\mathrm{H}} \mu=\inf \left\{\operatorname{dim}_{\mathrm{H}} A: A \in \mathcal{B}(X), \mu(A)=1\right\} .
$$

By a countable iterated function system with place dependent probabilities (briefly countable learning system) $(S, p)_{\aleph_{0}}=\left(S_{i}, p_{i}\right)_{i=0}^{\infty}$ we mean a sequence of continuous transformations $S_{i}: X \rightarrow X, i \in \mathbb{N}_{0}$, and a sequence of continuous functions $p_{i}: X \rightarrow[0,1], i \in \mathbb{N}_{0}$, such that $\sum_{i=0}^{\infty} p_{i}(x)=1$ for $x \in X$. The sequence $\left(p_{i}\right)_{i=0}^{\infty}$ as above is called a probability vector. We assume that $S_{i}$ is Lipschitzian with Lipschitz constant $L_{i}$ for $i \in \mathbb{N}_{0}$.

For a learning system $(S, p)_{\aleph_{0}}$ we define

$$
\lambda_{(S, p)_{\aleph_{0}}}=\max _{x \in X} \sum_{i=1}^{\infty} p_{i}(x) L_{i} .
$$

For a given learning system $(S, p)_{\aleph_{0}}$ we define the corresponding Markov operator $P_{(S, p)_{\aleph_{0}}}: \mathcal{M} \rightarrow \mathcal{M}$ by

$$
P_{(S, p)_{\aleph_{0}}} \mu(A)=\sum_{i=1}^{\infty} \int_{S_{i}^{-1}(A)} p_{i}(x) \mu(d x) \quad \text { for } A \in \mathcal{B}(X)
$$

and its adjoint $U_{(S, p)_{\aleph_{0}}}: C(X) \rightarrow C(X)$ by

$$
U_{(S, p)_{\aleph_{0}}} f(x)=\sum_{i=1}^{\infty} p_{i}(x) f\left(S_{i}(x)\right) \quad \text { for } x \in X .
$$

In this paper we consider continuous learning systems. Fix $K \in(0, \infty]$. Let $S:[0, K) \times X \rightarrow X$ be a continuous function. We assume that there exists a measurable function $\lambda_{S}:[0, K) \rightarrow \mathbb{R}_{+}$such that

$$
\|S(t, x)-S(t, y)\| \leq \lambda_{S}(t)\|x-y\| \quad \text { for } x, y \in X, t \in[0, K) .
$$

Let $p:[0, K) \times X \rightarrow \mathbb{R}_{+}$be a continuous function such that

$$
\int_{0}^{K} p(t, x) d t=1 \quad \text { for } x \in X
$$

We assume that there exists $c_{p}>0$ such that

$$
\int_{0}^{K}|p(t, x)-p(t, y)| d t \leq c_{p}\|x-y\|
$$

for every $x, y \in X$.

Every such pair $(S, p)$ will be called a continuous iterated function system with place dependent probabilities (briefly continuous learning system). The 
set of all continuous functions $p:[0, K) \rightarrow \mathbb{R}_{+}$satisfying (2.3) and (2.4) is denoted by $\mathbb{P}_{[0, K)}$.

Set

$$
\lambda_{(S, p)}=\max _{x \in X} \int_{0}^{K} p(t, x) \lambda_{S}(t) d t .
$$

A continuous learning system $(S, p)$ such that $\lambda_{(S, p)} \leq 1$ will be called nonexpansive.

For a given learning system $(S, p)$ we define the corresponding Markov operator $P_{(S, p)}: \mathcal{M} \rightarrow \mathcal{M}$ by

$$
P_{(S, p)} \mu(A)=\int_{X} \int_{0}^{K} \mathbb{1}_{A}(S(t, x)) p(t, x) d t \mu(d x) \quad \text { for } A \in \mathcal{B}(X)
$$

and its adjoint $U_{(S, p)}: C(X) \rightarrow C(X)$ by

$$
U_{(S, p)} f(x)=\int_{0}^{K} p(t, x) f(S(t, x)) d t \quad \text { for } x \in X .
$$

For two continuous learning systems $(S, p)$ and $(T, g)$ we define

$$
d((S, p),(T, q))=\widetilde{d}(p, q)+\bar{d}(S, T),
$$

where

$$
\begin{aligned}
\widetilde{d}(p, q) & =\sup _{x \in X}\left(\int_{0}^{K}|p(t, x)-q(t, x)| d t+\sup _{t \in[0, K)}|p(t, x)-q(t, x)|\right), \\
\bar{d}(S, T) & =\sup _{(t, x) \in[0, K) \times X}\|S(t, x)-T(t, x)\| .
\end{aligned}
$$

Denote by $\mathcal{F}$ the set of all continuous nonexpansive learning systems $(S, p)$. Observe that $\mathcal{F}$ endowed with the metric $d$ is a complete metric space.

We say that the learning system $(S, p)$ has a stationary distribution (resp. is asymptotically stable) if the corresponding Markov operator $P_{(S, p)}$ has a stationary distribution (resp. is asymptotically stable).

Finally, recall that a subset of a complete metric space $\mathcal{X}$ is called residual if its complement is a set of first Baire category.

\section{Auxiliary results}

Lemma 3.1. Let $\mu_{1}, \mu_{2} \in \mathcal{M}_{1}, l \geq 1$ and $\varepsilon>0$. If $\left\|\mu_{1}-\mu_{2}\right\|_{l} \leq \varepsilon^{2}$, then

$$
\mu_{1}(B(A, \varepsilon)) \geq \mu_{2}(A)-\varepsilon
$$

for every $A \in \mathcal{B}(X)$.

The lemma follows from [11, Lemma 3.1]. 
Lemma 3.2. If $q \in \mathbb{P}_{[0, K)}$, then for every $\varepsilon>0$ there exists a function $p \in \mathbb{P}_{[0, K)}$ such that

$$
p(t, x)>0 \quad \text { for } t \in[0, K), x \in X
$$

and

$$
\widetilde{d}(p, q)<\varepsilon .
$$

Proof. Fix $\varepsilon \in(0,1)$. Let $r, k:[0, K) \rightarrow \mathbb{R}_{+}$be continuous functions such that

$$
0<k(t) \leq \max \{1,1 / K\} \quad \text { for } t \in[0, K)
$$

(we admit $1 / \infty=0$ ),

$$
\int_{0}^{K} k(t) d t=1
$$

and

$$
1-\frac{\varepsilon}{4 \max \left\{\max _{x \in X} q(t, x), 1,1 / K\right\}}<r(t)<1 \quad \text { for } t \in[0, K) .
$$

We define the function $p:[0, K) \times X \rightarrow \mathbb{R}_{+}$by the formula

$$
p(t, x)=r(t) q(t, x)+k(t) \int_{0}^{K}(1-r(s)) q(s, x) d s
$$

for $(t, x) \in[0, K) \times X$. It is easy to see that $p$ is continuous and conditions (2.3) and (3.1) are satisfied. For every $x, y \in X$ we have

$$
\begin{aligned}
\int_{0}^{K}|p(t, x)-p(t, y)| d t \leq & \int_{0}^{K}|r(t) q(t, x)-r(t) q(t, y)| d t \\
& +\int_{0}^{K}|(1-r(s)) q(s, x)-(1-r(s)) q(s, y)| d s \\
= & \int_{0}^{K}|q(t, x)-q(t, y)| d t \leq c_{q}\|x-y\|
\end{aligned}
$$

and so (2.4) is satisfied with constant $c_{p}=c_{q}$. By (3.3) for $(t, x) \in[0, K) \times X$ we have

$$
\begin{aligned}
|p(t, x)-q(t, x)|=\mid & (r(t)-1) q(t, x)+k(t) \int_{0}^{K}(1-r(s)) q(s, x) d s \mid \leq \varepsilon / 2, \\
& \int_{0}^{K}|p(t, x)-q(t, x)| d t \leq \varepsilon / 2
\end{aligned}
$$

and (3.2) follows. 
Let $(S, p)_{\aleph_{0}}$ be an asymptotically stable countable learning system and let $\mu$ be the corresponding invariant distribution. Define

$$
X_{(S, p)_{\aleph_{0}}}=\{x \in X: \mu(\{x\})>0\} .
$$

Lemma 3.3. If $\mu\left(X_{(S, p)_{\aleph_{0}}}\right)>0$, then for every $\varepsilon>0$ there exists a finite set $Z_{\varepsilon} \subset X_{(S, p)_{\aleph_{0}}}$ such that $\mu\left(Z_{\varepsilon}\right)>1-\varepsilon$.

The proof can be found in [1, Corollary 3.1]. In fact, Corollary 3.1 in [1] was proved for finite learning systems but the same argument works for countable learning systems.

REMARK 3.1. Let $(S, p)$ be a continuous learning system such that

$$
\lambda_{(S, p)}<1 .
$$

Let $P_{(S, p)}$ and $U_{(S, p)}$ be the corresponding Markov operator and its adjoint, respectively. Then there exists $l \geq 1$ such that

$$
U_{(S, p)} f \in F_{l} \quad \text { for } f \in F_{l}
$$

and consequently $P_{(S, p)}$ is nonexpansive in the norm $\|\cdot\|_{l}$. Indeed, a simple calculation shows that (3.5) holds for

$$
l=\max \left\{\frac{c_{p}}{1-\lambda_{(S, p)}}, 1\right\}
$$

where $c_{p}$ is defined by (2.4).

LEMMA 3.4. If a continuous learning system $(S, p)$ satisfies (3.1) and (3.4), then it is asymptotically stable and for every $\varepsilon>0$ there exists $n \in \mathbb{N}$ such that

$$
\left\|P_{(S, p)}^{n} \mu_{1}-P_{(S, p)}^{n} \mu_{2}\right\|_{l}<\varepsilon \quad \text { for } \mu_{1}, \mu_{2} \in \mathcal{M}_{1},
$$

where $l$ is defined in Remark 3.1.

Proof. We claim that for every $\varepsilon>0$ there are a Borel set $A$ with $\operatorname{diam} A \leq \varepsilon$, a real number $\alpha>0$ and an integer $m$ such that

$$
P_{(S, p)}^{m} \mu(A) \geq \alpha \quad \text { for } \mu \in \mathcal{M}_{1} .
$$

In fact, fix $\varepsilon>0$. From (3.4) it follows that there exists $E \subset[0, K)$ such that $l^{1}(E)>0$ and

$$
\gamma=\sup _{t \in E} \lambda_{S}(t)<1,
$$

where $l^{1}$ stands for one-dimensional Lebesgue measure. Now we can choose $a \in(0, K]$ such that for every $\delta>0$,

$$
l^{1}([a, a+\delta] \cap E)>0 .
$$


Let $m \in \mathbb{N}$ be such that $\gamma^{m} \operatorname{diam} X \leq \varepsilon / 2$. From (2.2) it follows that there exists $\delta_{1}>0$ such that

$$
\operatorname{diam}\left(\bigcup_{t_{1} \in\left[a, a+\delta_{1}\right] \cap E} S\left(t_{1}, X\right)\right) \leq \gamma \operatorname{diam} X+\frac{\gamma \varepsilon}{2 m} .
$$

By an induction argument for every $k \in\{1, \ldots, m\}$ we can find $\delta_{k}>0$ such that

$$
\begin{aligned}
& \operatorname{diam}\left(\bigcup_{t_{k} \in E_{k}} S\left(t_{k}, \bigcup_{t_{k-1} \in E_{k-1}} S\left(t_{k-1}, \ldots \bigcup_{t_{1} \in E_{1}} S\left(t_{1}, X\right) \ldots\right)\right)\right) \\
& \quad \leq \gamma \operatorname{diam}\left(\bigcup_{t_{k-1} \in E_{k-1}} S\left(t_{k-1}, \ldots \bigcup_{t_{1} \in E_{1}} S\left(t_{1}, X\right) \ldots\right)\right)+\frac{\gamma^{k} \varepsilon}{2 m} \\
& \quad \leq \gamma^{k}\left(\operatorname{diam} X+\frac{k \varepsilon}{2 m}\right)
\end{aligned}
$$

where $E_{i}=\left[a, a+\delta_{i}\right] \cap E$. Define

$$
A=\bigcup_{\left\{t_{1}, \ldots, t_{m}\right\} \subset[a, a+\delta] \cap E} S\left(t_{m}, S\left(t_{m-1}, \ldots, S\left(t_{1}, X\right) \ldots\right)\right),
$$

where $\delta=\min \left\{\delta_{1}, \ldots, \delta_{m}\right\}$. Clearly diam $A \leq \varepsilon$. From the definition of the adjoint Markov operator we have

$$
\begin{aligned}
P_{(S, p)}^{m} \mu(A) & =\int_{X} \int_{0}^{K} \ldots \int_{0}^{K} p\left(t_{1}, x\right) \ldots p\left(t_{m}, S\left(t_{m-1}, \ldots S\left(t_{1}, x\right)\right)\right) \\
& \times \mathbb{1}_{A}\left(S\left(t_{m}, \ldots S\left(t_{1}, x\right)\right)\right) d t_{1} \ldots d t_{m} \mu(d x) \\
& \geq \int_{X([a, a+\delta] \cap E)^{m}} \int_{\ldots \int_{1}} p\left(t_{1}, x\right) \ldots p\left(t_{m}, S\left(t_{m-1}, \ldots S\left(t_{1}, x\right)\right)\right) \\
& \times \mathbb{1}_{A}\left(S\left(t_{m}, \ldots S\left(t_{1}, x\right)\right)\right) d t_{1} \ldots d t_{m} \mu(d x) \\
& \left.\geq l^{1}([a, a+\delta] \cap E)\right)^{m} \underset{(t, x) \in[a, a+\delta] \cap E \times X}{\inf }(p(t, x))^{m}>0
\end{aligned}
$$

for every $\mu \in \mathcal{M}_{1}$. This completes the proof of the claim. Application of the proof of Theorem 3.1 in [9] finishes the proof.

The following lemma is an analogue of Lemma 3.2 in [8].

LEMMA 3.5. If a continuous learning system $(S, p) \in \mathcal{F}$ satisfies condition (3.4), then for all $\varepsilon>0$ and $n \in \mathbb{N}$ there exists $\delta>0$ such that for each $(T, q) \in \mathcal{F}$

$$
d((S, p),(T, q))<\delta \Rightarrow\left(\left\|P_{(S, p)}^{n} \mu-P_{(T, q)}^{n} \mu\right\|_{l}<\varepsilon \text { for } \mu \in \mathcal{M}_{1}\right),
$$

where $l$ is defined by Remark 3.1 . 
Proof. Fix $\varepsilon>0, n \in \mathbb{N}$. Let $f \in F_{l}$ and $x \in X$. Then

$$
\begin{aligned}
\mid U_{(S, p)} & f(x)-U_{(T, q)} f(x) \mid \\
& =\left|\int_{0}^{K} p(t, x) f(S(t, x)) d t-\int_{0}^{K} q(t, x) f(T(t, x)) d t\right| \\
& \leq \int_{0}^{K}|p(t, x)-q(t, x)| d t+\int_{0}^{K} q(t, x)|f(S(t, x))-f(T(t, x))| d t \\
& \leq \int_{0}^{K}|p(t, x)-q(t, x)| d t+l \int_{0}^{K} q(t, x)\|S(t, x)-T(t, x)\| d t \\
& \leq \widetilde{d}(p, q)+l \bar{d}(S, T) .
\end{aligned}
$$

This yields

$$
\left|U_{(S, p)} f(x)-U_{(T, q)} f(x)\right| \leq l d((S, p),(T, q)) .
$$

For $m>1$ we have

$$
\begin{aligned}
\left|U_{(S, p)}^{m} f(x)-U_{(T, q)}^{m} f(x)\right| \leq & \left|U_{(T, q)}\left(U_{(S, p)}^{m-1} f\right)(x)-U_{(T, q)}\left(U_{(T, q)}^{m-1} f\right)(x)\right| \\
& +\left|U_{(T, q)}\left(U_{(S, p)}^{m-1} f\right)(x)-U_{(S, p)}\left(U_{(S, p)}^{m-1} f\right)(x)\right| \\
\leq & \sup _{y \in X}\left|U_{(S, p)}^{m-1} f(y)-U_{(T, q)}^{m-1} f(y)\right| \\
& +\left|U_{(T, q)}\left(U_{(S, p)}^{m-1} f\right)(x)-U_{(S, p)}\left(U_{(S, p)}^{m-1} f\right)(x)\right| .
\end{aligned}
$$

Since $U_{(S, p)}^{m-1} f \in F_{l}$, we obtain

$$
\begin{aligned}
\left|U_{(S, p)}^{m} f(x)-U_{(T, q)}^{m} f(x)\right| \leq & \sup _{h \in F_{l}, y \in X}\left|U_{(S, p)}^{m-1} h(y)-U_{(T, q)}^{m-1} h(y)\right| \\
& +\sup _{h \in F_{l}, y \in X}\left|U_{(S, p)} h(y)-U_{(T, q)} h(y)\right| .
\end{aligned}
$$

This and inequality (3.8) yield

$$
\begin{gathered}
\sup _{f \in F_{l}, x \in X}\left|U_{(S, p)}^{m} f(x)-U_{(T, q)}^{m} f(x)\right| \leq \sup _{f \in F_{l}, x \in X}\left|U_{(S, p)}^{m-1} f(x)-U_{(T, q)}^{m-1} f(x)\right| \\
+l d((S, p),(T, q)) .
\end{gathered}
$$

Consequently, we have

$$
\begin{aligned}
\left\|P_{(S, p)}^{n} \mu-P_{(T, q)}^{n} \mu\right\|_{l} & \leq \sup _{f \in F_{l}, x \in X}\left|U_{(S, p)}^{n} f(x)-U_{(T, q)}^{n} f(x)\right| \\
& \leq n l d((S, p),(T, q))
\end{aligned}
$$

for $\mu \in \mathcal{M}_{1}$. Set $\delta=\varepsilon(n l)^{-1}$ to complete the proof. 
For every $n \in \mathbb{N}$, let $\mathcal{F}_{n}$ be the set of all $(S, p) \in \mathcal{F}$ satisfying (3.1) and (3.4) and such that there exist $Z_{n} \subset X$ and $r_{n} \in(0,1 / n)$ such that

$$
\mu_{(S, p)}\left(B\left(Z_{n}, r_{n}\right)\right)>1-1 / n
$$

and

$$
\mu_{(S, p)}\left(B\left(x, 4 r_{n}\right)\right) \geq 3 r_{n}^{1 / n} / 2 \quad \text { for } x \in B\left(Z_{n}, 2 r_{n}\right),
$$

where $\mu_{(S, p)}$ is the stationary distribution corresponding to $(S, p)$.

Lemma 3.6. For every $n \in \mathbb{N}$ the set $\mathcal{F}_{n}$ is dense in the space $(\mathcal{F}, d)$.

Proof. Let $z \in X$ and $\alpha \in(0,1)$. Since $X$ is convex, for every transformation $T:[0, K) \times X \rightarrow X$ we can define a transformation $\widehat{T}:[0, K) \times X$ $\rightarrow X$ by

$$
\widehat{T}(t, x)=\alpha z+(1-\alpha) T(t, x) \quad \text { for }(t, x) \in[0, K) \times X .
$$

From this and Lemma 3.2 it follows that the set $\widehat{\mathcal{F}}$ of all learning systems $(S, p) \in \mathcal{F}$ satisfying (3.1) and (3.4) is dense in $\mathcal{F}$. To complete the proof it is sufficient to show that for every $n \in \mathbb{N}$, the set $\mathcal{F}_{n}$ is dense in $\widehat{\mathcal{F}}$.

Fix $n \in \mathbb{N},(T, p) \in \widehat{\mathcal{F}}$ and $\varepsilon>0$. Since $X$ is compact and $T:[0, K) \times X \rightarrow$ $X$ is continuous, we can choose two sequences $\left(a_{i}\right)_{i \in \mathbb{N}_{0}}$ and $\left(b_{i}\right)_{i \in \mathbb{N}_{0}}$ such that

$$
\begin{gathered}
a_{0}=0, \quad a_{i-1}<a_{i}<b_{i-1} \leq a_{i+1} \quad \text { for } i \in \mathbb{N}, \\
{[0, K)=\bigcup_{i=1}^{\infty}\left[a_{i}, b_{i}\right]}
\end{gathered}
$$

and

$$
\|T(t, x)-T(\bar{t}, x)\|<\varepsilon / 2 \quad \text { for } t, \bar{t} \in\left[a_{i-1}, b_{i-1}\right], i \in \mathbb{N}, x \in X .
$$

Let $s_{0}=0$ and $s_{i} \in\left(a_{i}, b_{i-1}\right)$ for $i \in \mathbb{N}$. Define $\gamma=\left(1-\lambda_{(T, p)}\right) / 2$. For every $i \in \mathbb{N}_{0}$ we can choose $\bar{t}_{i} \in\left[s_{i}, s_{i+1}\right)$ such that

$$
\lambda_{T}\left(\bar{t}_{i}\right) \leq \lambda_{T}(t)+\gamma \quad \text { for } t \in\left[s_{i}, s_{i+1}\right)
$$

and consequently

$$
\lambda_{T}\left(\bar{t}_{i}\right) \int_{s_{i}}^{s_{i+1}} p(t, x) d t \leq \int_{s_{i}}^{s_{i+1}}\left(\lambda_{T}(t)+\gamma\right) p(t, x) d t
$$

for $x \in X$. For $i \in \mathbb{N}_{0}$ we define $\widetilde{S}_{i}: X \rightarrow X$ by

$$
\widetilde{S}_{i}(x)=T\left(\bar{t}_{i}, x\right)
$$

and $\widetilde{S}:[0, K) \times X \rightarrow X$ by

$$
\widetilde{S}(t, x)=\widetilde{S}_{i}(x) \quad \text { for }(t, x) \in\left[s_{i}, s_{i+1}\right) \times X .
$$


From $\left[s_{i}, s_{i+1}\right) \subset\left(a_{i}, b_{i}\right)$ and inequality (3.11) it follows that

$$
\sup _{(t, x) \in[0, K) \times X}\|\widetilde{S}(t, x)-T(t, x)\| \leq \varepsilon / 2 .
$$

Observe that for every $i \in \mathbb{N}_{0}$ the function $\widetilde{S}_{i}$ is Lipschitzian with Lipschitz constant $L_{i}=\lambda_{T}\left(\bar{t}_{i}\right)$. Consider a countable learning system $(\widetilde{S}, p)_{\aleph_{0}}=$ $\left(\widetilde{S}_{i}, p_{i}\right)_{i=0}^{\infty}$ where $\widetilde{S}_{i}$ is given by $(3.12)$ and

$$
p_{i}(x)=\int_{s_{i}}^{s_{i+1}} p(t, x) d t .
$$

We have

$$
\begin{aligned}
\lambda_{(\widetilde{S}, p)_{\aleph_{0}}} & =\max _{x \in X} \sum_{i=1}^{\infty} L_{i} p_{i}(x)=\max _{x \in X} \sum_{i=1}^{\infty} \lambda_{T}\left(\bar{t}_{i}\right) \int_{s_{i}}^{s_{i}+1} p(t, x) d t \\
& \leq \max _{x \in X} \sum_{i=0}^{\infty} \int_{s_{i}}^{s_{i+1}}\left(\lambda_{T}(t)+\gamma\right) p(t, x) d t \\
& =\max _{x \in X} \int_{0}^{K}\left(\lambda_{T}(t)+\gamma\right) p(t, x) d t=\lambda_{(T, p)}+\gamma<1 .
\end{aligned}
$$

Consequently, there exists $j_{0} \in \mathbb{N}_{0}$ such that $L_{j_{0}}<1$. Let $x_{0} \in X$ be a fixed point of $\widetilde{S}_{j_{0}}$. Choose $\delta$ such that

$$
0<\delta<\min \left\{1-L_{j_{0}},\left(1-\lambda_{(\widetilde{S}, p)_{\aleph_{0}}}\right)\left(\max _{x \in X} p_{j_{0}}(x)\right)^{-1}\right\} .
$$

By [5, Lemma 3.5] there exists $r>0$ and a Lipschitz transformation $\widetilde{S}: X \rightarrow X$ with Lipschitz constant $L_{\widetilde{S}}$ and with the following properties:

$$
\begin{gathered}
L_{\widetilde{S}}<L_{j_{0}}+\delta<1, \\
\max _{x \in X}\left\|\widetilde{S}(x)-\widetilde{S}_{j_{0}}(x)\right\|<\varepsilon / 2, \\
\widetilde{S}(x)=x_{0} \quad \text { for }\left\|x-x_{0}\right\| \leq r .
\end{gathered}
$$

Consider a countable learning system $(\widehat{S}, p)_{\aleph_{0}}=\left(\widehat{S}_{i}, p_{i}\right)_{i=0}^{\infty}$ such that

$$
\widehat{S}_{i}(x)= \begin{cases}\widetilde{S}(x) & \text { for } i=j_{0}, \\ \widetilde{S}_{i}(x) & \text { for } i \neq j_{0} .\end{cases}
$$

Set $\lambda_{\widehat{S}}=\lambda_{(\widehat{S}, p)_{\aleph_{0}}}$. Since $\lambda_{\widehat{S}}<1$, the system $(\widehat{S}, p)_{\aleph_{0}}$ is asymptotically stable (see [3]). From (3.14), (3.16) and compactness of $X$ it follows that there exists an integer $m$ such that

$$
\widehat{S}_{j_{0}}^{m}(x)=x_{0} \quad \text { for } x \in X .
$$


Let $P_{\widehat{S}}$ and $\mu_{\widehat{S}}$ be the Markov operator corresponding to $(\widehat{S}, p)_{\aleph_{0}}$ and its invariant distribution, respectively. We have

$$
\begin{aligned}
\mu_{\widehat{S}}\left(\left\{x_{0}\right\}\right)= & P_{\widehat{S}}^{m} \mu_{\widehat{S}}\left(\left\{x_{0}\right\}\right) \\
= & \sum_{i_{1}, \ldots, i_{m}=1}^{\infty} \int_{X} p_{i_{1}}(x) \ldots\left(p_{i_{m}} \circ S_{i_{m-1}} \circ \cdots \circ S_{i_{1}}\right)(x) \\
& \quad \times \mathbb{1}_{\left\{x_{0}\right\}}\left(\widehat{S}_{i_{1}} \circ \cdots \circ \widehat{S}_{i_{m}}\right)(\{x\}) \mu_{\widehat{S}}(d x) .
\end{aligned}
$$

Consequently,

$$
\mu_{\widehat{S}}\left(\left\{x_{0}\right\}\right) \geq\left(\min _{x \in X} p_{j_{0}}(x)\right)^{m} \mu_{\widehat{S}}(X)>0
$$

From this and Lemma 3.3 it follows that there exists a finite set $Z_{n} \subset X$ such that

$$
\mu_{\widehat{S}}\left(Z_{n}\right)>1-\frac{1}{2 n}
$$

and

$$
\mu_{\widehat{S}}(x)>0 \quad \text { for } x \in Z_{n} .
$$

Let $r_{n} \in(0,1 / n)$ be such that

$$
\mu_{\widehat{S}}\left(B\left(x, r_{n}\right)\right) \geq 2 r_{n}^{1 / n} \quad \text { for } x \in Z_{n} .
$$

Set $\widehat{l}=\max \left\{2 c_{p} /\left(1-\lambda_{\widehat{S}}\right), 1\right\}$. Let $m \in \mathbb{N}$ be such that

$$
L_{\widehat{S}_{j_{0}}}^{m} \operatorname{diam} X \leq \frac{r_{n}^{2}}{32 \widehat{l}}
$$

and let $k \in \mathbb{N}$ be such that

$$
\left(1-\left(\min _{(t, x) \in\left[0, s_{j_{0}+1}\right] \times X} p(t, x)\right)^{m}\right)^{k} \leq \frac{r_{n}^{2}}{32} .
$$

Since $X$ is compact we can choose a sequence $\left(t_{i}\right)_{i \in \mathbb{N}}$ such that $t_{i} \in\left(a_{i}, s_{i}\right)$ for $i \in \mathbb{N}$ and the following properties are satisfied:

$$
\begin{aligned}
& \max _{x \in X} \sum_{i=1}^{\infty}\left(L_{\widehat{S}_{i-1}}+L_{\widehat{S}_{i}}\right) \int_{t_{i}}^{s_{i}} p(t, x) d t<\frac{1-\lambda_{\widehat{S}}}{2}, \\
& \max _{x \in X} \sum_{i=1}^{\infty} \int_{t_{i}}^{s_{i}} p(t, x) d t \leq \frac{\left(1-\lambda_{\widehat{S}}\right) r_{n}^{2}}{16 m k\left(c_{p}+1\right) \operatorname{diam} X} .
\end{aligned}
$$

Now, we define the function $S:[0, K) \times X \rightarrow X$ by the formula $S(t, x)= \begin{cases}\frac{t-t_{i}}{s_{i}-t_{i}} \widehat{S}_{i}(x)+\frac{s_{i}-t}{s_{i}-t_{i}} \widehat{S}_{i-1}(x) & \text { for }(t, x) \in\left(t_{i}, s_{i}\right) \times X, i \in \mathbb{N}, \\ \widehat{S}_{i-1}(x) & \text { for }(t, x) \in\left[s_{i-1}, t_{i}\right] \times X, i \in \mathbb{N} .\end{cases}$ 
It is easy to check that $S(t, x)$ is continuous and

$$
\sup _{(t, x) \in[0, K) \times X}\|S(t, x)-T(t, x)\| \leq \varepsilon .
$$

By (3.20) we have

$$
\begin{aligned}
\lambda_{(S, p)} & =\max _{x \in X} \int_{0}^{K} \lambda_{S}(t) p(t, x) d t \\
& =\max _{x \in X}\left(\sum_{i=0}^{\infty} \int_{s_{i}}^{t_{i+1}} \lambda_{S}(t) p(t, x) d t+\sum_{i=1}^{\infty} \int_{t_{i}}^{s_{i}} \lambda_{S}(t) p(t, x) d t\right) \\
& \leq \max _{x \in X}\left(\sum_{i=0}^{\infty} L_{\widehat{S}_{i}} \int_{s_{i}}^{t_{i+1}} p(t, x) d t+\sum_{i=1}^{\infty}\left(L_{\widehat{S}_{i-1}}+L_{\widehat{S}_{i}}\right) \int_{t_{i}}^{s_{i}} p(t, x) d t\right) \\
& <\lambda_{\widehat{S}}+\frac{1-\lambda_{\widehat{S}}}{2}<1 .
\end{aligned}
$$

Consequently, due to Lemma $3.4,(S, p)$ is asymptotically stable. Moreover, there exists an interval $[a, b] \subset\left[0, s_{j_{0}+1}\right]$ such that

$$
\lambda_{S}(t) \leq L_{\widehat{S}_{j_{0}}} \quad \text { for } t \in[a, b] .
$$

Let $P_{(S, p)}, U_{(S, p)}$ and $\mu$ be the Markov operator corresponding to $(S, p)$, its dual and the invariant distribution, respectively. Let $l$ be the constant defined by Remark 3.1. Since $\widehat{l} \geq l$, from (3.18), (3.22) and the proof of Lemma 3.4 it follows that there exists a Borel set $A$ such that (3.7) holds for $\alpha=\left(\min _{(t, x) \in[a, b] \times X} p(t, x)\right)^{m}$ and $\operatorname{diam} A \leq r_{n}^{2}(16 l)^{-1}$. From this, (3.19) and the proof of Theorem 3.1 in [9] it follows that (3.6) holds for $n=m k$ and $\varepsilon=r_{n}^{2} / 8$. Hence

$$
\begin{aligned}
\left\|\mu_{\widehat{S}}-\mu\right\|_{l} & =\left\|P_{\widehat{S}}^{m k} \mu_{\widehat{S}}-P_{(S, p)}^{m k} \mu\right\|_{l} \\
& \leq\left\|P_{\widehat{S}}^{m k} \mu_{\widehat{S}}-P_{(S, p)}^{m k} \mu_{\widehat{S}}\right\|_{l}+\left\|P_{(S, p)}^{m k} \mu_{\widehat{S}}-P_{(S, p)}^{m k} \mu\right\|_{l} \\
& \leq\left\|P_{\widehat{S}}^{m k} \mu_{\widehat{S}}-P_{(S, p)}^{m k} \mu_{\widehat{S}}\right\|_{l}+r_{n}^{2} / 8 .
\end{aligned}
$$

Fix $f \in F_{l}$. Using the definition of $S(t, x)$ we obtain

$$
\begin{aligned}
\left|U_{\widehat{S}}^{m k} f(x)-U_{(S, p)}^{m k} f(x)\right| & \\
\leq & \sum_{i=1}^{\infty} \int_{s_{i-1}}^{s_{i}} p(t, x)\left|U_{\widehat{S}}^{m k-1} f\left(\widehat{S}_{i-1}(x)\right)-U_{(S, p)}^{m k-1} f(S(t, x))\right| d t \\
\quad= & \sum_{i=1}^{\infty} \int_{t_{i}}^{s_{i}} p(t, x)\left|U_{\widehat{S}}^{m k-1} f\left(\widehat{S}_{i-1}(x)\right)-U_{(S, p)}^{m k-1} f(S(t, x))\right| d t
\end{aligned}
$$




$$
\begin{aligned}
& +\sum_{i=1}^{\infty} \int_{s_{i-1}}^{t_{i}} p(t, x)\left|U_{\widehat{S}}^{m k-1} f\left(\widehat{S}_{i-1}(x)\right)-U_{(S, p)}^{m k-1} f\left(\widehat{S}_{i-1}(x)\right)\right| d t \\
\leq & \sum_{i=1}^{\infty} \int_{t_{i}}^{s_{i}} p(t, x)\left|U_{\widehat{S}}^{m k-1} f\left(\widehat{S}_{i-1}(x)\right)-U_{(S, p)}^{m k-1} f(S(t, x))\right| d t \\
& +\sum_{i=1}^{\infty} \int_{s_{i-1}}^{t_{i}} p(t, x)\left(\sum_{j=1}^{\infty} \int_{s_{j-1}}^{s_{j}} p(s, x) \mid U_{\widehat{S}}^{m k-2} f\left(\widehat{S}_{j-1}\left(\widehat{S}_{i-1}(x)\right)\right)\right. \\
& \left.-U_{(S, p)}^{m k-2} f\left(S\left(s,\left(\widehat{S}_{i-1}(x)\right)\right)\right) \mid d s\right) d t \\
\leq & \sum_{i=1}^{\infty} \int_{t_{i}}^{s_{i}} p(t, x)\left|U_{\widehat{S}}^{m k-1} f\left(\widehat{S}_{i-1}(x)\right)-U_{(S, p)}^{m k-1} f(S(t, x))\right| d t \\
& +\sum_{i=1}^{\infty} p_{i}(x)\left(\sum_{j=1}^{\infty} \int_{t_{j}}^{s_{j}} p(s, x) \mid U_{\widehat{S}}^{m k-2} f\left(\widehat{S}_{j-1}\left(\widehat{S}_{i-1}(x)\right)\right)\right. \\
& \left.-U_{(S, p)}^{m k-2} f\left(S\left(s, \widehat{S}_{i-1}(x)\right)\right) \mid d s\right) \\
& +\sum_{i=1}^{\infty} p_{i}(x)\left(\sum_{j=1}^{\infty} \int_{s_{j-1}}^{t_{j}} p(s, x) \mid U_{\widehat{S}}^{m k-2} f\left(\widehat{S}_{j-1}\left(\widehat{S}_{i-1}(x)\right)\right)\right. \\
& \left.-U_{(S, p)}^{m k-2} f\left(\widehat{S}_{j-1}\left(\widehat{S}_{i-1}(x)\right)\right) \mid d s\right)
\end{aligned}
$$

for $x \in X$. Consequently, by an induction argument we have

$$
\left|U_{\widehat{S}}^{m k} f(x)-U_{(S, p)}^{m k} f(x)\right| \leq m k l \operatorname{diam} X \cdot \sum_{i=1}^{\infty} \int_{t_{i}}^{s_{i}} p(t, x) d t
$$

for $x \in X$. Hence

$$
\begin{aligned}
\left\|P_{\widehat{S}}^{m k} \mu_{\widehat{S}}-P_{(S, p)}^{m k} \mu_{\widehat{S}}\right\|_{l} & =\sup _{f \in F_{l}}\left|\left\langle f, P_{\widehat{S}}^{m k} \mu_{\widehat{S}}\right\rangle-\left\langle f, P_{(S, p)}^{m k} \mu_{\widehat{S}}\right\rangle\right| \\
& =\sup _{f \in F_{l}}\left|\left\langle U_{\widehat{S}}^{m k} f, \mu_{\widehat{S}}\right\rangle-\left\langle U_{(S, p)}^{m k} f, \mu_{\widehat{S}}\right\rangle\right| \\
& \leq \sup _{f \in F_{l}}\left\langle m k l \operatorname{diam} X \cdot \sup _{x \in X} \sum_{i=1}^{\infty} \int_{t_{i}}^{s_{i}} p(t, x) d t, \mu_{\widehat{S}}\right\rangle \\
& \leq m k \widehat{l} \operatorname{diam} X \cdot \sup _{x \in X} \sum_{i=1}^{\infty} \int_{t_{i}}^{s_{i}} p(t, x) d t .
\end{aligned}
$$

By (3.21) this gives

$$
\left\|P_{\widehat{S}}^{m k} \mu_{\widehat{S}}-P_{(S, p)}^{m k} \mu_{\widehat{S}}\right\|_{l} \leq r_{n}^{2} / 8
$$


From this and (3.23) it follows that

$$
\left\|\mu_{\widehat{S}}-\mu\right\|_{l} \leq r_{n}^{2} / 8+r_{n}^{2} / 8=r_{n}^{2} / 4
$$

Moreover, for every $y \in B\left(Z_{n}, 2 r_{n}\right)$ there exists $x \in Z_{n}$ such that

$$
B\left(x, r_{n}\right) \subset B\left(y, 3 r_{n}\right) .
$$

Consequently, by (3.17) and Lemma 3.1 we obtain

$$
\begin{aligned}
\mu\left(B\left(y, 4 r_{n}\right)\right) & \geq \mu_{\widehat{S}}\left(B\left(y, 3 r_{n}\right)\right)-r_{n} / 2 \geq \mu_{\widehat{S}}\left(B\left(x, r_{n}\right)\right)-r_{n} / 2 \\
& \geq 2 r_{n}^{1 / n}-r_{n}^{1 / n} / 2=3 r_{n}^{1 / n} / 2 .
\end{aligned}
$$

From the definition of $Z_{n}$ and Lemma 3.1 it follows that

$$
\mu\left(B\left(Z_{n}, r_{n}\right)\right) \geq \mu_{\widehat{S}}\left(Z_{n}\right)-r_{n} / 2 \geq 1-1 / 2 n-1 / 2 n=1-1 / n .
$$

The proof is complete.

LEMMA 3.7. Assume that $(S, p)$ is an asymptotically stable continuous learning system and $\mu$ is the corresponding invariant measure. Let $\alpha \geq 0$. If

$$
\mu\left(\left\{x \in X: \liminf _{r \rightarrow 0} \frac{\log \mu(B(x, r))}{\log r} \leq \alpha\right\}\right)=1,
$$

then $\operatorname{dim}_{\mathrm{H}} \mu \leq \alpha$.

The proof can be found in [1, Lemma 3.8]. In fact, Lemma 3.8 in [1] was proved for finite learning systems but the same argument works for continuous learning systems.

\section{Main theorem}

THEOREM 4.1. The set $\widehat{\mathcal{F}}_{*}$ of all $(T, q) \in \mathcal{F}$ such that the unique invariant distribution $\mu_{(T, q)}$ satisfies $\operatorname{dim}_{\mathrm{H}} \mu_{(T, q)}=0$, is residual in $\mathcal{F}$.

Proof. Fix $n \in \mathbb{N}$ and $(S, p) \in \mathcal{F}_{n}$. Let $P_{(S, p)}$ be the Markov operator corresponding to $(S, p)$ and let $\mu_{(S, p)}$ be its stationary distribution. Let $Z_{(S, p), n} \subset X$ and $r_{(S, p), n} \leq 1 / n$ be such that

$$
\mu_{(S, p)}\left(B\left(Z_{(S, p), n}, r_{(S, p), n}\right)\right)>1-1 / n
$$

and

$$
\mu_{(S, p)}\left(B\left(x, 4 r_{(S, p), n}\right)\right) \geq 3 r_{(S, p), n}^{1 / n} / 2 \quad \text { for } x \in B\left(Z_{(S, p), n}, 2 r_{(S, p), n}\right) .
$$

Let $l_{(S, p)} \geq 1$ be defined by Remark 3.1. By Lemma 3.4 there exists $k_{(S, p), n}$ $\in \mathbb{N}$ such that

$$
\left\|P_{(S, p)}^{k_{(S, p), n}} \mu_{1}-P_{(S, p)}^{k_{(S, p), n}} \mu_{2}\right\|_{l_{(S, p)}} \leq r_{(S, p), n}^{2} / 8 \quad \text { for } \mu_{1}, \mu_{2} \in \mathcal{M}_{1} .
$$

By Lemma 3.5 there exists $\delta_{(S, p), n}>0$ such that for all $(T, q) \in \mathcal{F}$,

$$
\begin{aligned}
d((S, p),(T, q)) & <\delta_{(S, p), n} \\
& \Rightarrow \sup _{\mu \in \mathcal{M}_{1}}\left\|P_{(S, p)}^{k_{(S, p), n}} \mu-P_{(T, q)}^{k_{(S, p), n}} \mu\right\|_{l_{(S, p)}} \leq r_{(S, p), n}^{2} / 8 .
\end{aligned}
$$


Define

$$
\widehat{\mathcal{F}}=\bigcap_{n=1}^{\infty} \bigcup_{(S, p) \in \mathcal{F}_{n}} B_{\mathcal{F}}\left((S, p), \delta_{(S, p), n}\right),
$$

where $B_{\mathcal{F}}\left((S, p), \delta_{(S, p), n}\right)$ is the open ball in $(\mathcal{F}, d)$ with center $(S, p)$ and radius $\delta_{(S, p), n}$. From Lemma 3.6 it follows that $\widehat{\mathcal{F}}$ is an intersection of countably many open dense sets. Consequently, $\widehat{\mathcal{F}}$ is residual. We are going to show that $\widehat{\mathcal{F}} \subset \widehat{\mathcal{F}}_{*}$.

Fix $(T, q) \in \widehat{\mathcal{F}}$. Let $P_{(T, q)}$ denotes the corresponding Markov operator. Since $X$ is compact, the operator $P_{(T, q)}$ has an invariant distribution $\mu_{(T, q)}$ (see [6]). From (4.3) and (4.4) it follows that $(T, q)$ is asymptotically stable (see the proof of Theorem 4.1 in [1]).

Let $\left((S, p)_{n}\right)_{n \in \mathbb{N}}$ be a sequence of learning systems such that $(S, p)_{n} \in \mathcal{F}_{n}$ and

$$
(T, q) \in B_{\mathcal{F}}\left((S, p)_{n}, \delta_{(S, p)_{n}, n}\right) \quad \text { for } n \in \mathbb{N} .
$$

For every $n \in \mathbb{N}$ assume that $Z_{(S, p)_{n}, n} \subset X, r_{(S, p)_{n}, n} \in(0,1 / n)$, $l_{(S, p)_{n}, n}>1$ and $k_{(S, p)_{n}, n} \in \mathbb{N}$ are such that (4.1)-(4.3) hold for the corresponding operator $P_{(S, p), n}$ and its stationary measure $\mu_{(S, p), n}$. For abbreviation we set

$$
\begin{array}{rlrl}
P_{n} & =P_{(S, p), n}, & \mu_{n}=\mu_{(S, p), n}, & Z_{n}=Z_{(S, p)_{n}, n} \\
r_{n}=r_{(S, p)_{n}, n}, & l_{n}=l_{(S, p), n}, & k_{n}=k_{(S, p)_{n}, n} .
\end{array}
$$

By (4.3) and (4.4) we obtain

$$
\begin{aligned}
\left\|\mu_{(T, q)}-\mu_{n}\right\|_{l_{n}} & =\left\|P_{(T, q)}^{k_{n}} \mu_{(T, q)}-P_{n}^{k_{n}} \mu_{n}\right\|_{l_{n}} \\
& \leq\left\|P_{(T, q)}^{k_{n}} \mu_{(T, q)}-P_{n}^{k_{n}} \mu_{(T, q)}\right\|_{l_{n}}+\left\|P_{n}^{k_{n}} \mu_{(T, q)}-P_{n}^{k_{n}} \mu_{n}\right\|_{l_{n}} \\
& \leq r_{n}^{2} / 4 .
\end{aligned}
$$

Consequently, by (4.2) and Lemma 3.1 for every $x \in B\left(Z_{n}, 2 r_{n}\right)$ we have

$$
\mu_{(T, q)}\left(B\left(x, 5 r_{n}\right)\right) \geq \mu_{n}\left(B\left(x, 4 r_{n}\right)\right)-r_{n} / 2 \geq 3 r_{n}^{1 / n} / 2-r_{n}^{1 / n} / 2=r_{n}^{1 / n} .
$$

Define

$$
Y=\bigcap_{m=1}^{\infty} \bigcup_{n=m}^{\infty} B\left(Z_{n}, 2 r_{n}\right)
$$

By the definition of $Z_{n}$ and Lemma 3.1 we have

$$
\mu_{(T, q)}\left(B\left(Z_{n}, 2 r_{n}\right)\right) \geq \mu_{n}\left(B\left(Z_{n}, r_{n}\right)\right)-r_{n} \geq 1-1 / n-1 / n=1-2 / n .
$$

Consequently, $\mu_{(T, q)}(Y)=1$. On the other hand, if $y \in Y$ then $y \in B\left(Z_{n}, 2 r_{n}\right)$ for infinitely many $n \in \mathbb{N}$ and by $(4.5)$ we can choose a sequence $\left(s_{n}\right)_{n \in \mathbb{N}}$ of integers such that

$$
\mu_{(T, q)}\left(B\left(y, 5 r_{s_{n}}\right)\right) \geq r_{s_{n}}^{1 / s_{n}} \quad \text { for } n \in \mathbb{N}
$$


Hence

$$
\lim _{n \rightarrow \infty} \frac{\log \mu_{(T, q)}\left(B\left(y, 5 r_{s_{n}}\right)\right)}{\log 5 r_{s_{n}}} \leq \lim _{n \rightarrow \infty} \frac{\log r_{s_{n}}^{1 / s_{n}}}{\log 5 r_{s_{n}}}=0 .
$$

Since $y \in Y$ was arbitrary, by Lemma 3.7 we have $\operatorname{dim}_{\mathrm{H}} \mu_{(T, q)}=0$. The proof is complete.

\section{References}

[1] T. Bielaczyc, Generic properties of iterated function systems with place dependent probabilities, Univ. Iagel. Acta Math. 44 (2006), 35-46.

[2] R. M. Dudley, Probabilities and Metrics, Aarhus Universitet, 1976.

[3] P. Janoska, Stability of a countable iterated function system, Univ. Iagel. Acta Math. 32 (1995), 105-119.

[4] A. Lasota and M. C. Mackey, Cell division and the stability of cellular populations, J. Math. Biol. 38 (1999), 241-261.

[5] A. Lasota and J. Myjak, Generic properties of fractal measures, Bull. Polish Acad. Sci. Math. 42 (1994), 283-296.

[6] A. Lasota and J. A. Yorke, Lower bound technique for Markov operators and iterated function systems, Random. Comput. Dynam. 2 (1994), 41-77.

[7] T. Szarek, Generic properties of continuous iterated function systems, Bull. Polish Acad. Sci. Math. 57 (1999), 77-89.

[8] —, Generic properties of learning systems, Ann. Polon. Math. 73 (2000), 93-102.

[9] - Iterated function systems depending on a previous transformation, Univ. Iagel. Acta Math. 33 (1996), 161-172.

[10] -, On typical Markov operators acting on Borel measures, Abstr. Appl. Anal. 5 (2005), 489-497.

[11] - The stability of Markov operators on Polish spaces, Studia Math. 143 (2000), $145-152$.

Tomasz Bielaczyc

Institute of Mathematics

Silesian University

40-007 Katowice, Poland

E-mail: bielaczyc@ux2.math.us.edu.pl

Received April 14, 2005;

received in final form February 27, 2007 